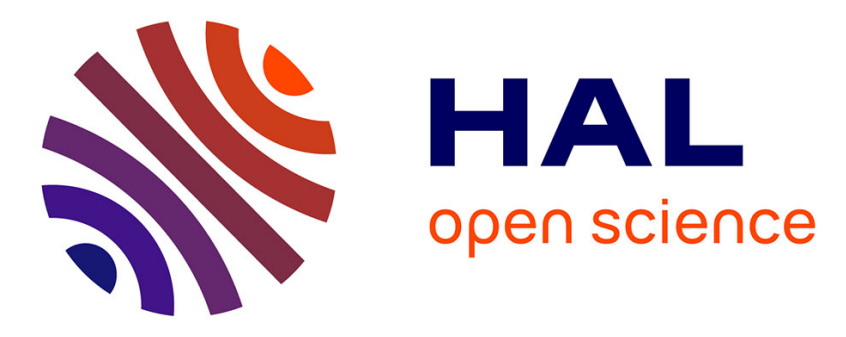

\title{
Vehicle yaw control via coordinated use of steering/braking systems
}

\author{
Moustapha Doumiati, Olivier Sename, John Jairo Martinez Molina, Luc \\ Dugard, Peter Gaspar, Zoltan Szabo, Jozsef Bokor
}

\section{- To cite this version:}

Moustapha Doumiati, Olivier Sename, John Jairo Martinez Molina, Luc Dugard, Peter Gaspar, et al.. Vehicle yaw control via coordinated use of steering/braking systems. IFAC WC 2011 - 18th IFAC World Congress, Aug 2011, Milan, Italy. pp.644-649. hal-00681332

\section{HAL Id: hal-00681332 \\ https://hal.science/hal-00681332}

Submitted on 21 Mar 2012

HAL is a multi-disciplinary open access archive for the deposit and dissemination of scientific research documents, whether they are published or not. The documents may come from teaching and research institutions in France or abroad, or from public or private research centers.
L'archive ouverte pluridisciplinaire HAL, est destinée au dépôt et à la diffusion de documents scientifiques de niveau recherche, publiés ou non, émanant des établissements d'enseignement et de recherche français ou étrangers, des laboratoires publics ou privés. 


\title{
Vehicle yaw control via coordinated use of steering/braking systems
}

\author{
M. Doumiati, O. Sename, J. Martinez, L. Dugard* \\ P. Gaspar, Z. Szabo, J. Bokor ** \\ * Gipsa-Lab UMR CNRS 5216, Control Systems Department, Grenoble \\ Institute of Technology ENSE3, BP 46, 38402 Saint Martin d'Hères, \\ France (olivier.sename@gipsa-lab.grenoble-inp.fr) \\ ** Computer and Automation Research Institute, Hungarian Academy \\ of Sciences, Kende u. 13-17, H-1111, Budapest, Hungary \\ (gaspar@sztaki.hu)
}

\begin{abstract}
The aim of this paper is to present a novel methodology that deals with steering/braking coordination task for vehicle yaw control. For steerability enhancement, only active steering control is involved. However, when the vehicle reaches the handling limits, both steering and braking collaborate together to ensure vehicle stability. Judging the vehicle stability region is deduced from the phase-plane of the sideslip angle and its time derivative. The coordination of the steering/braking actuators is achieved through a suitable gain scheduled $L P V$ (Linear Parameter Varying) controller. The controller is synthesized within the LMI (Linear Matrix Inequalities) framework, while warranting $\mathcal{H}_{\infty}$ performances. The simulation results show the effectiveness of the proposed control scheme when the vehicle is subject to various driving situations.
\end{abstract}

\section{INTRODUCTION}

Under critical driving circumstances, it is difficult for a driver to stabilize the vehicle, and dangerous accidents could happen. Safety of ground vehicles requires the improvement of yaw stability by active control. The basic idea is to assist the vehicle handling to be close to a linear vehicle handling characteristic that is familiar to the driver, and to restrain the vehicle lateral dynamics to be within the stable handling region in aggressive maneuvers.

By applying additional steering angle to front/rear wheels, Active Steering control $(A S)$ can enhance steerability and lateral vehicle dynamics by directly regulating tire slip angles and thus the lateral tire forces (Guven et al. [2007], Rajamani [2006]). Unfortunately, $A S$ becomes less effective when the vehicle reaches the handling limits due to the tire saturations. In order to maintain vehicle stability under critical driving conditions, an alternative approach utilizes differential braking forces between the left and the right sides of the vehicle to produce the required corrective yaw moment (Rajamani [2006], Park [2001], Boada et al. [2005]). This braking based technique is referred to as Direct Yaw moment Control $(D Y C)$. Note that $D Y C$ is effective in both vehicle linear/nonlinear region, however, it is not desirable in normal conditions because of the direct influence of the control action on the longitudinal vehicle dynamics (i.e it causes the vehicle to slow down significantly). Consequently, these two control techniques are optimized individually in specific handling regions, and the maximum benefit can be gained through the coordinated use of both methods of corrective yaw motion generation in the control strategy. In this field, several solutions have been proposed in recent years ( $\mathrm{He}$ et al. [2006] and Yang et al. [2009]), and this topic is still an object of intense research activities from both industrial and academic sides. It is worth noting that some studies also involve suspension actuators to improve handling performances, like in (Poussot-Vassal et al. [2010]), but this is out of the scope of this paper.

This work deals with the design of a new vehicle chassis control scheme that integrates and coordinates braking and front steering in order to enhance vehicle handling and yaw stability. The proposed control design is worked out on the basis of a 2-DOF (Degree-Of-Freedom) linear planar vehicle model, and it is formulated as a Linear Parameter Varying $(L P V)$ controller structure. The exogenous scheduling control parameter is function of the sideslip angle dynamics, and it activates the braking control only in critical maneuvers. The controller is synthesized within the Linear Matrix Inequalities $(L M I)$ framework, while guaranting $\mathcal{H}_{\infty}$ performances.

This new control methodology constitutes the major contribution of this paper. It aims at simplifying engineer design, reducing development time in making actuators cooperate, guaranteeing robustness properties with respect to model uncertainties, and internal stability. Besides, the proposed strategy does not involve any online optimization process, that means it could be suitable for real-time applications.

The response of the vehicle with the proposed control scheme has been evaluated with MATLAB computer simulations using a complex full vehicle nonlinear model subject to various driving situations. The obtained results confirm the effectiveness of the proposed control.

The rest of this paper is structured as follows. Section II first introduces the global control scheme, and then develops and synthesizes the vehicle dynamical stability controller. Performance analysis is done in Section III through time simulations. Conclusions and discussions are given in Section IV.

\section{Paper notations:}

Throughout the paper, the following notations will be 


\begin{tabular}{llcl}
\hline Symbol & Value & Unit & Signification \\
\hline \hline$m$ & 1535 & $\mathrm{~kg}$ & vehicle mass \\
$m_{r}$ & 648 & $\mathrm{~kg}$ & vehicle rear mass \\
$I_{z}$ & 2149 & $\mathrm{~kg} \cdot \mathrm{m}^{2}$ & vehicle yaw inertia \\
$C_{f}$ & 40000 & $\mathrm{~N} / \mathrm{rd}$ & cornering stiffness of front tires \\
$C_{r}$ & 40000 & $\mathrm{~N} / \mathrm{rd}$ & cornering stiffness of rear tires \\
$l_{f}$ & 1.4 & $\mathrm{~m}$ & distance COG - front axle \\
$l_{r}$ & 1 & $\mathrm{~m}$ & distance COG - rear axle \\
$t_{r}$ & 1.4 & $\mathrm{~m}$ & rear axle length \\
$h$ & 0.5 & $\mathrm{~m}$ & height of the center of gravity \\
\hline$\mu$ & {$[2 / 5 ; 1]$} & - & tire/road contact friction interval \\
$v$ & {$[50 ; 130]$} & $\mathrm{km} / \mathrm{h}$ & vehicle velocity interval \\
$g$ & 9.81 & $\mathrm{~m} / \mathrm{s}^{2}$ & gravitational acceleration \\
\hline
\end{tabular}

Table 1. Notations and vehicle parameters.

adopted: index $i=\{f, r\}$ and $j=\{l, r\}$ are used to identify vehicle front, rear and left, right positions respectively. Table 1 summarizes the vehicle parameters, notations and values used in the paper.

\section{CONTROL SYSTEM DESIGN}

The control system is shown in the block diagram of figure 1. The main goal of the proposed control system is to make the actual yaw rate, $\dot{\psi}$, to follow the desired yaw rate, $\dot{\psi}_{d}$. The reference model is adopted to keep the vehicle within the linear region that is familiar to the driver. Another purpose is to limit the vehicle sideslip angle, $\beta$, to be within an acceptable region to prevent vehicle spin.

As seen in figure 1, the proposed controller responds to yaw rate error, and its outputs are the active steer angle and the wheel brake torque. In the following, each block of the control scheme will be described in details.

\subsection{Reference model}

To obtain a desired vehicle response, it is necessary that the yaw rate follows its target value $\dot{\psi}_{d}$. In this work, $\dot{\psi}_{d}$ is calculated using a 2-DOF classical linear bicycle model depicted in figure 2. The use of this model is explained in details in (Dugoff et al. [1970]). Roll, pitch, and longitudinal dynamics are neglected to simplify the vehicle dynamics. The equations governing the lateral and yaw motions in this model can be expressed as:

- Equation of lateral motion:

$$
m v(\dot{\beta}-\dot{\psi})=C_{f}\left(\delta-\beta-l_{f} \frac{\dot{\psi}}{v}\right)+C_{r}\left(-\beta+l_{r} \frac{\dot{\psi}}{v}\right)
$$

- Equation of yaw motion:

$$
I_{z} \ddot{\psi}=C_{f}\left(-l_{f} \frac{\dot{\psi}}{v}-\beta-\delta\right) l_{f}+C_{r}\left(\beta-l_{r} \frac{\dot{\psi}}{v}\right) l_{r}
$$

Consequently, $\dot{\psi}_{d}$ is function of the driver steering wheel angle, $\delta_{d}$, and the vehicle speed, $v$.

Since the lateral acceleration of the vehicle cannot exceed the maximum friction coefficient $\mu$, the desired yaw rate must be limited by the following value (Rajamani [2006]):

$$
\left|\dot{\psi}_{d, \max }\right| \leq|\mu g / v|
$$

\subsection{Yaw controller}

The function of the yaw control system is to restore the yaw rate of the vehicle as close as possible to the

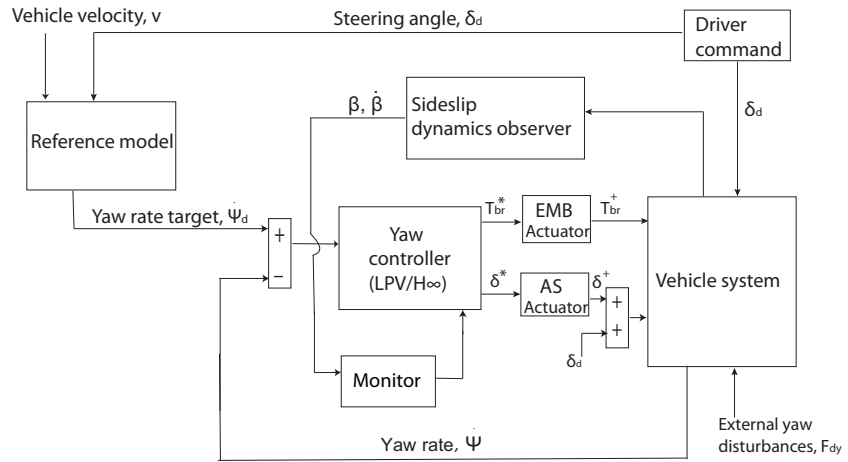

Fig. 1. Global control scheme.

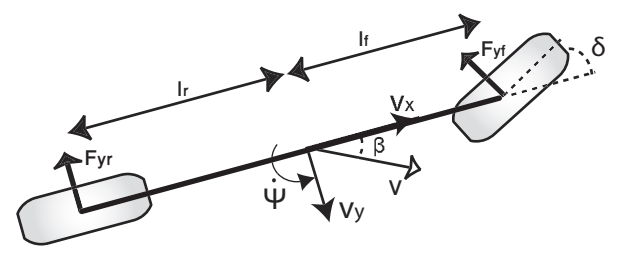

Fig. 2. 2-DOF model of lateral vehicle dynamics.

nominal motion expected by the driver. Consequently, the controller is designed so that the vehicle follows the reference yaw rate through driving the tracking error between the actual and desired yaw rate to zero.

The controller structure is hierarchical and designed in 2 layers:

a) The upper controller provides the active steer angle, $\delta^{*}$, and the corrective yaw moment, $M_{z}^{*}$, needed to track the target yaw rate, and thus ensures the vehicle handling. Besides, it is supposed to reject disturbances that may affect the lateral motion of the vehicle.

Note that when the vehicle is in its linear region, the controller ensures steerability, and only steering is used to follow the desired response. However, when the vehicle reaches the handling limits, steering and braking act together to maintain the vehicle stability.

b) The lower controller converts the stabilizing yaw moment generated by the upper controller into effective braking torque, and it decides which wheel must be braked to counteract the undesired yaw motion.

a) Upper controller: $L P V / \mathcal{H}_{\infty}$ controller design

The 2-DOF linear bicycle model is used as the control model. Although the bicycle model is relatively simple, it includes the important features of the lateral vehicle dynamics. Taking into account the controller structure, this model is extended to include: 1 ) the direct yaw moment input $M_{z}^{*}$, and 2) a lateral disturbance force $F_{d y}$. In the following, the extended linear bicycle model given in system (4) is used for synthesis. To synthesize the upper control, the $\mathcal{H}_{\infty}$ control performance is used. For more information about the $\mathcal{H}_{\infty}$ theory, reader can refer to Skogestad et al. [2007]. In the following, the generalized synthesis plant together with the performance weighting functions, called here $\sum_{g}$, is presented and illustrated in figure 3. Dynamics of the actuators are neglected during the controller design process. $\sum_{g}$ is given thereafter: 


$$
\left[\begin{array}{c}
\ddot{\psi} \\
\dot{\beta}
\end{array}\right]=\left[\begin{array}{cc}
-\frac{l_{f}^{2} C_{f}+l_{r}^{2} C_{r}}{I_{z} v} & \frac{l_{r} C_{r}-l_{f} C_{f}}{I_{z}} \\
1+\frac{l_{r} C_{r}-l_{f} C_{f}}{m v^{2}} & -\frac{C_{f}+C_{r}}{m v}
\end{array}\right]\left[\begin{array}{c}
\dot{\psi} \\
\beta
\end{array}\right]+\left[\begin{array}{c}
\frac{l_{f} C_{f}}{I_{z}} \\
\frac{C_{f}}{m v}
\end{array}\right] \delta^{*}+\left[\begin{array}{c}
\frac{1}{I_{z}} \\
0
\end{array}\right] M_{z}^{*}+\left[\begin{array}{c}
0 \\
\frac{1}{m v}
\end{array}\right] F_{d y}
$$

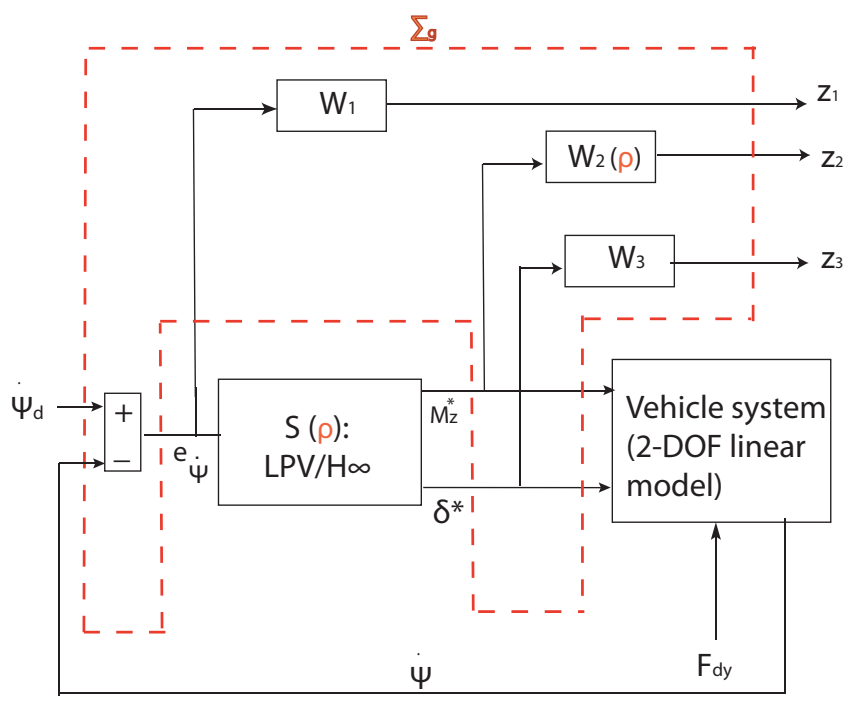

Fig. 3. Generalized plant model for synthesis.

$$
\Sigma_{g}:\left[\begin{array}{c}
\dot{x} \\
z \\
y
\end{array}\right]=\left[\begin{array}{ccc}
A & B_{1} & B_{2} \\
C_{1} & D_{11} & D_{12} \\
C_{2} & 0 & 0
\end{array}\right]\left[\begin{array}{l}
x \\
w \\
u
\end{array}\right]
$$

where $x$ includes the state variables of the system and of the weighting functions, $w=\left[\dot{\psi}_{d}, F_{d y}\right]^{T}$ is the exogenous input vector, $u=\left[\delta^{*}, M_{z}^{*}\right]^{T}$ represents the control input signals, $y=\left[e_{\dot{\psi}}\right]^{T}$ is the measurement vector, and $z=\left[z_{1}, z_{2}, z_{3}\right]^{T}$ contains the weighted controlled outputs which have to be as small as possible.

\section{Weighting functions:}

In order to formulate the standard structure for the $\mathcal{H}_{\infty}$ controller defined in figure 3 , the weighting functions $W_{1}$, $W_{2}$, and $W_{3}$ are defined to characterize the performance objectives and the actuator limitations (actuator descriptions are given in Subsection 2.4):

- $W_{1}$ weights the yaw rate error signal:

$$
W_{1}=7 \frac{s / 10 \pi f_{1}+1}{s / 2 \pi f_{1}+1} .
$$

where $f_{1}=8 \mathrm{~Hz}$ is the cut-off frequency of the low pass filter. $W_{1}$ is shaped in order to reduce the yaw rate error.

- $W_{2}$ weights the braking control signal according to a scheduling parameter $\rho$ :

$$
W_{2}=\rho \times 10^{-3} \frac{s / 70 f_{2}+1}{s / 700 f_{2}+1},
$$

where $f_{2}=10 \mathrm{~Hz}$ is the braking actuator cut-off frequency. $W_{2}$ is linearly parameterized by the considered varying parameter $\rho($.$) , where \rho \in\{\underline{\rho} \leq \rho \leq \bar{\rho}\}$ (with $\rho=0.1$ and $\bar{\rho}=10$ ). Then, when $\rho=\bar{\rho}$, the braking input is penalized, on the contrary, when $\rho=\rho$, the braking control signal is relaxed.

- $W_{3}$ weights the steering control signal:

$$
\begin{aligned}
W_{3} & =G_{\delta}^{0} \frac{\left(s / 2 \pi f_{3}+1\right)\left(s / 2 \pi f_{4}+1\right)}{\left(s / \alpha 2 \pi f_{4}+1\right)^{2}} \\
G_{\delta}^{0} & =\frac{\left(\Delta_{f} / \alpha 2 \pi f_{4}+1\right)^{2}}{\left(\Delta_{f} / 2 \pi f_{3}+1\right)\left(\Delta_{f} / 2 \pi f_{4}+1\right)} \\
\Delta_{f} & =2 \pi\left(f_{4}+f_{3}\right) / 2,
\end{aligned}
$$

where $f_{3}=1 \mathrm{~Hz}$ is the lower limit of the actuator intervention, and $f_{4}=10 \mathrm{~Hz}$ is the steering actuator bandwidth. This filter is designed in order to allow the steering control to act only in $\left[f_{3}, f_{4}\right]$ frequency range, where the driver cannot act. Thus, it is ensured that the steering action is comfortable for the driver. This filter design is inspired from Guven et al. [2007].

These weighting functions are recalled thereafter in the sensitivity function plots as upper bounds limits, $1 / W_{i}$ (see figure 5).

\section{Problem resolution: $L M I$ based $L P V / \mathcal{H}_{\infty}$ :}

The $\mathcal{H}_{\infty}$ problem consists in finding a stabilizing controller, named $S(\rho)$ (see figure 3), scheduled by $\rho$, of the form:

$$
S(\rho):\left[\begin{array}{c}
\dot{x}_{c} \\
u
\end{array}\right]=\left[\begin{array}{cc}
A_{c}(\rho) & B_{c}(\rho) \\
C_{c}(\rho) & 0
\end{array}\right]\left[\begin{array}{c}
x_{c} \\
y
\end{array}\right]
$$

that minimizes the $\mathcal{H}_{\infty}$ norm of the closed-loop $L P V$ system formed by the interconnection of equations (5) and (9), where $u=\left[\delta^{*}, M_{z}^{*}\right]^{T}$ and $y=\left[e_{\dot{\psi}}\right]^{T}$.

As the parameter dependency, $\rho$, enters in a linear way in the system definition, the $L P V$ polytopic approach solution consists in finding a common Lyapunov function at each vertex $\{\underline{\rho}, \bar{\rho}\}$, of the polytope defined by system (5). Thus, a $L M I$ problem has to be solved, minimizing the attenuation level $\gamma$ (Scherer et al. [1997]). Using Yalmip/Sedumi solver (Lofberg [2004], Sturm [1999]), one obtains the optimal attenuation level $\gamma_{\text {opt }}=0.89$. Then the applied controller is a convex combination of the controllers synthesized at the vertices $\{\rho, \bar{\rho}\}$ (for more details about the polytopic approach, refer to Apkarian et al. [1995]).

Figure 4 shows the steering and braking controller output according to $\rho$. As the braking weight has been described as parameter dependent, it is shown that when $\rho=10$ braking signal is attenuated, and conversely, when $\rho=0.1$ braking gain is larger. As a consequence, when $\rho$ is low (resp. high), braking is activated (resp. disabled). Intermediate values will give intermediate behaviors. Remember that, for any $\rho \in\{\underline{\rho} \leq \rho \leq \bar{\rho}\}$, the closed loop stability is guaranted thanks to the $L P V$ design and the polytopic approach.

According to the sensitivity functions Bode diagrams illustrated in figure 5, it is interesting to make the following deductions:

- $\frac{e_{\psi}}{\dot{\psi}_{d}}$ emphasizes that the yaw rate tracking performance satisfies the required specifications (see figure $5(\mathrm{a}))$

- The braking control is activated for $\rho=0.1$, and it is disabled for $\rho=10$ (see figure 5(b)). Note that intermediate values of $\rho \in[\underline{\rho}, \bar{\rho}]$ will give intermediate behaviors. Recall that, thanks to the $L P V$ polytopic 

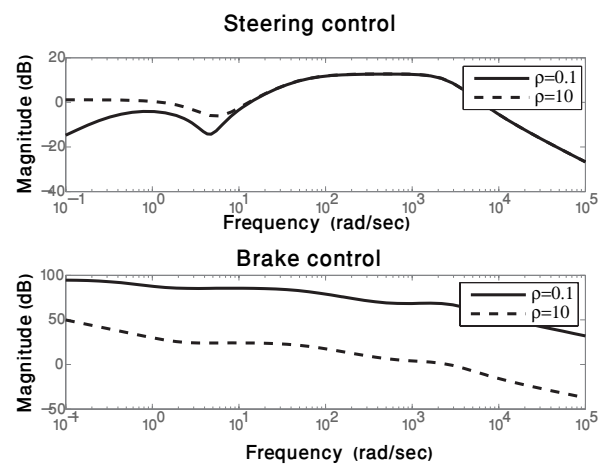

Fig. 4. Bode diagrams of the controller outputs $\delta^{*}$ and $M_{z}^{*}$.

approach, the closed loop stability is guaranteed for any $\rho \in[\rho, \bar{\rho}]$.

- The steering control is activated especially in the specified frequency range $[1 \mathrm{~Hz}, 10 \mathrm{~Hz}]$ where the driver cannot act (see figure $5(\mathrm{c})$ ).

b) Lower controller: braking control scheme

The desired yaw moment $M_{z}^{*}$ determined by the upper controller can be generated by applying brake torque to appropriate wheels. In this study, to avoid overlapping with front steering actuators, only rear wheels are concerned in the control law:

$$
T_{b r j}^{*}=\left|\frac{2 R M_{z}^{*}}{t_{r}}\right|
$$

In the following, we develop the control law of the lower controller (assuming counterclockwise positive).

There are two situations that accompany yaw instability:

- for the understeer condition $\left(|\dot{\psi}| \prec\left|\dot{\psi}_{d}\right|\right)$, the control moment, $M_{z}^{*}$, is generated by applying braking torque on the inner rear wheel.

- for the oversteer condition $\left(|\dot{\psi}| \succ\left|\dot{\psi}_{d}\right|\right)$, the control yaw moment, $M_{z}^{*}$, is generated by applying braking torque on the outer rear wheel.

\subsection{Monitor: coordination strategy of steering and braking}

As the brake-based DYC technique is not desirable in normal driving situations because of its direct influence on the longitudinal dynamics, the aim of the monitor is to minimize the usage of the braking. Consequently, the braking actuators must only be used when the vehicle goes toward instability. Since vehicle stability is directly related to the sideslip motion of the vehicle, judging the vehicle stability region is derived from the phase-plane $(\beta-\dot{\beta})$. A stability bound defined in He et al. [2006] is used here, that is formulated as:

$$
\chi<1,
$$

where $\chi=|2.49 \dot{\beta}+9.55 \beta|$ is the "Stability Index".

Moreover, the control task is also supposed to provide a seamless introduction of the direct yaw moment control, when it is required. Hence, the scheduling parameter $\rho(\chi)$ can be defined as:
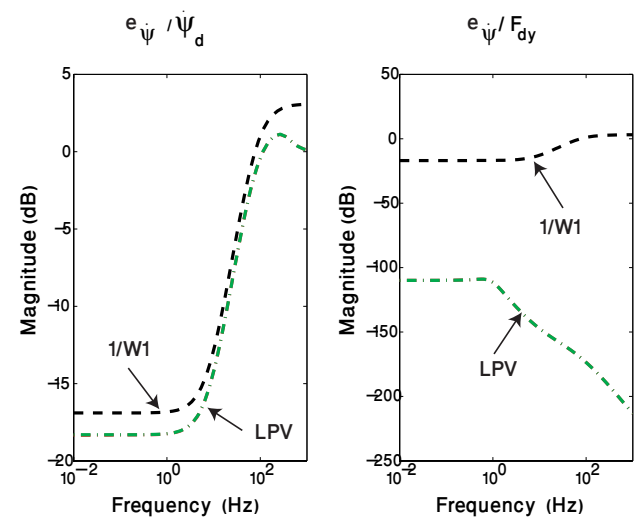

(a) Closed loop transfer functions between $e_{\dot{\psi}}$ and exogenous inputs.
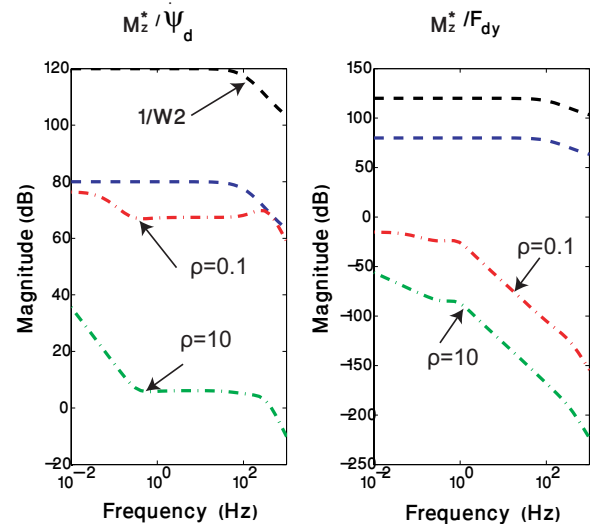

(b) Closed loop transfer functions between $M^{*}$ and exogenous inputs.
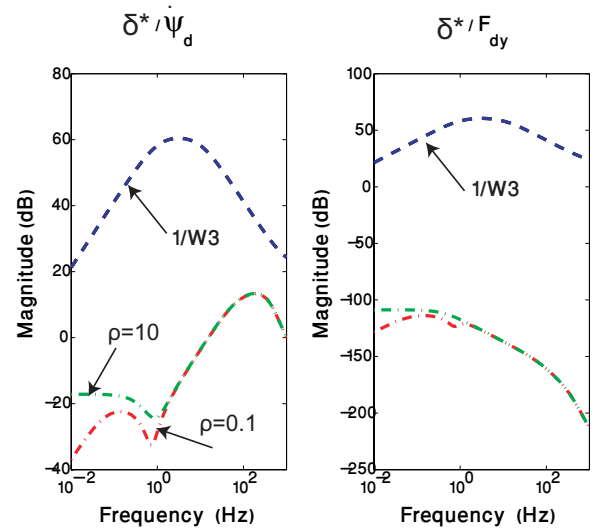

(c) Closed loop transfer functions between $\delta^{*}$ and exogenous inputs.

Fig. 5. Closed loop transfer functions: $L P V$ (red dashed $(\rho=0.1)$, or green dashed $(\rho=10))$ synthesis results; Inverse of weighting functions (black dashed) of $1 / W_{1}$, $1 / W_{2}, 1 / W_{3}$.

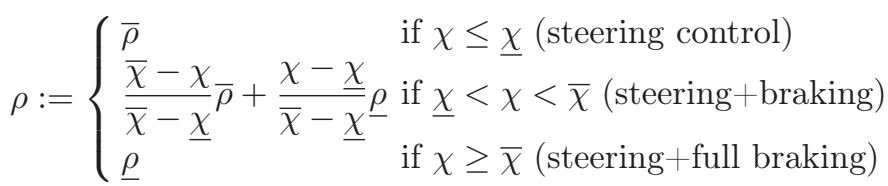

where $\chi=0.8$ ( $\chi$ is user defined) and $\bar{\chi}=1$. To calculate the actual stability index $\chi$, the block called "Sideslip 
dynamics observer" (see figure 3 ) evaluates $\dot{\beta}$ and $\beta$ in real-time:

- $\dot{\beta}$ can be reconstructed using available sensors, according to the following relationship:

$$
\dot{\beta}=\frac{a_{y}}{v_{x}}-\dot{\psi},
$$

where $a_{y}$ is the lateral acceleration, and $v_{x}$ is approximated by the mean of the rear wheel velocities.

- $\beta$ is not available using standard sensors, and thus, it must be estimated. " $\beta$-estimation" is widely discussed in the literature, and many papers deal with that topic (see You et al. [2009]). This estimation problem was also studied by the authors in a previous publication (Doumiati et al. [2010]).

\subsection{Actuator models}

The corrective steer angle and rear braking torques control signals can be generated via actuation systems. In this particular research, let us consider the following actuators:

- Steer-by-wire Active Steering (AS) system providing an additional steering angle. This actuator is modeled as:

$$
\dot{\delta}^{+}=2 \pi \kappa\left(\delta^{*}-\delta^{+}\right)
$$

where, $\kappa=10 \mathrm{~Hz}$ is the actuator cut-off frequency, $\delta^{*}$ and $\delta^{+}$are the steering controller and actuator outputs respectively. This actuator is bounded between $\left[-5^{\circ},+5^{\circ}\right]$.

- Brake-by-wire Electro Mechanical Braking (EMB) actuators providing a continuously variable braking torque. The EMB model is given by:

$$
\dot{T}_{b_{r j}}^{+}=2 \pi f_{2}\left(T_{b_{r j}}^{*}-T_{b_{r j}}^{+}\right)
$$

where, $f_{2}=10 \mathrm{~Hz}$ is the actuator cut-off frequency, $T_{b_{r j}}^{*}$ and $T_{b_{i j}}^{+}$are the local braking controller and actuator outputs respectively. This actuator control is limited between $[0,1200] \mathrm{Nm}$.

\subsection{Full vehicle model}

The block labelled "Vehicle simulation model" represents a full nonlinear vehicle model validated on a real "Renault Mégane Coupé" car. This model will be used in simulation for validation purpose only. Note that the main interest in using the full vehicle model is that it allows to take into account nonlinear load transfer, suspension dynamics, slipping and sideslip angles that are essential factors which play a major role on the global chassis dynamics, especially in critical driving situations. For more information about the vehicle model, see Poussot-Vassal et al. [2010].

\section{SIMULATION RESULTS AND ANALYSIS}

To clarify the effects of the proposed control scheme, both the vehicle dynamics with and without controllers are checked and compared. Different simulations were carried out on the nonlinear vehicle model platform briefly introduced in Subsection 2.5. Due to space limitations, only a double-lane-change test on a dry road $(\mu=0.9)$ is reported in this paper.

The vehicle is driven at $100 \mathrm{~km} / \mathrm{h}$. Figure 6 shows the yaw rate response versus the steering input. We can deduce that the uncontrolled vehicle becomes rather unstable as the amplitude of the steering input becomes larger. On the other hand, the controlled output of the yaw rate

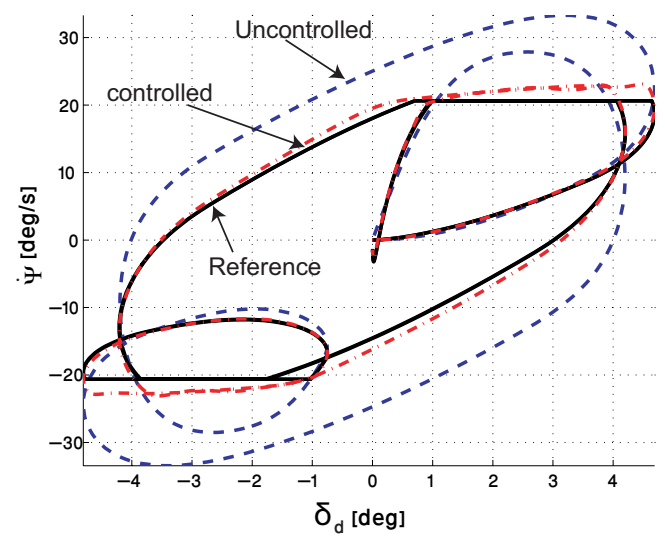

Fig. 6. Response of the yaw rates versus steering wheel angle: Controlled (red dash-dot), Uncontrolled (blue dot), Reference model (black solid)

has nearly converged to the output of the desired linear model. Comparisons between the sideslip angles and the trajectories of the uncontrolled and controlled vehicles are illustrated in figure 7 . Note that the sideslip angle is not specifically controlled in this study, it is simply the effect of the yaw rate control. According to these results, it is obvious that the handling performances are much improved by the proposed controller.

Figure 8 illustrates how the stability index and the dependency parameter $\rho$ evolve according to the driving situations. As stated before, when the stability index, $\chi$, is below 0.8 , only steering control is involved to enhance the handling performances. Therefore, $\rho$ is equal to 10 and the corrective yaw moment is penalized. On the other hand, when $\chi$ exceeds 0.8 , the braking system starts to work with the active steering to keep the vehicle stable. When $\chi$ becomes superior to $1, \rho$ takes the value 0.1 , and braking is fully activated.

Figure 9 shows the generated corrective steering angle and the brake torques to enhance the lateral vehicle control. It is worth noting, that despite this test agressivity, actuators are far from saturation that may lead to instability.

\section{CONCLUSION}

The focus of this work is on presenting the vehicle yaw stabilizing problem in the framework of a control scheme. The intent is to enhance vehicle steerability and stability during dynamic vehicle handling maneuvers. In this context, a new $L P V / \mathcal{H}_{\infty}$ controller that coordinates steering and braking actuators, is designed in this report. This $L P V$ controller is designed in an original way and ensures that: 1)Steerability is enhanced in normal driving condition, and 2) Braking is used only when the vehicle tends to instability. Simulations of critical driving situations that compare the responses of a controlled vehicle with respect to a passive vehicle show the effectiveness of the proposed control design.

Future work may consist to implement suspension actuators in the control scheme to enhance stability and prevent rollover situations.

\section{ACKNOWLEDGMENTS}

This work is supported by the national french project INOVE/ ANR 2010 BLAN 0308. 

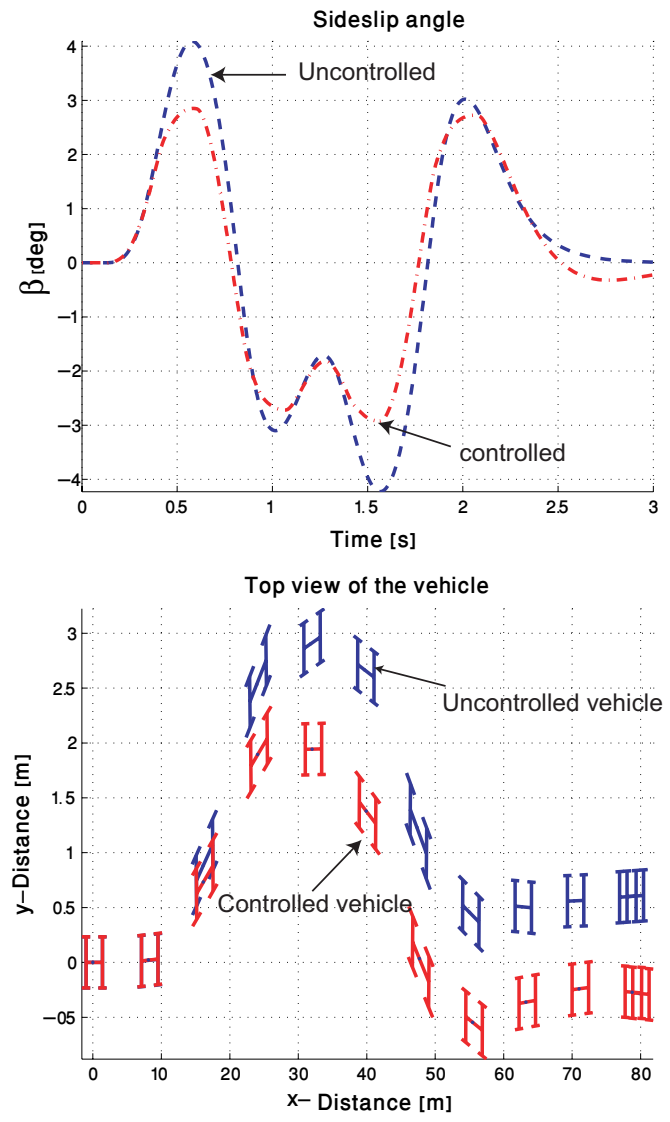

Fig. 7. Sideslip angles and trajectories of the controlled and uncontrolled vehicles: Controlled (red dash-dot), Uncontrolled (blue dash)
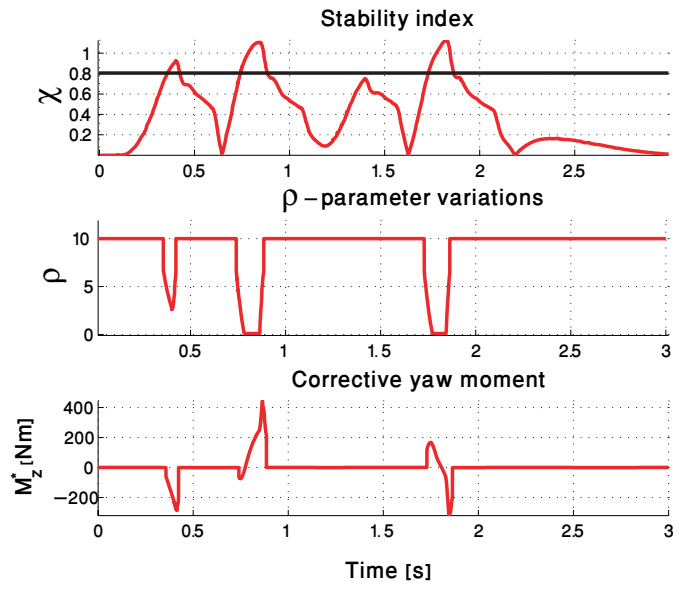

Fig. 8. $M_{z}^{*}$ and $\rho$ variations according to $\chi$ for the double lane-change maneuver.

\section{REFERENCES}

B. A. Guven, T. Bunte, D. Odenthal, and L. Guven. Robust two degree-of-freedom vehicle steering controller design IEEE Transaction on Control System Technology, volume 15, pages 554-565, 2007.

R. Rajamani. Vehicle dynamics and control. Springer, 2006.

J. H. Park. $H_{\infty}$ direct yaw-moment control with brakes for robust performance and stability of vehicles. JSME International Journal, series C, volume 44(2), 2001.
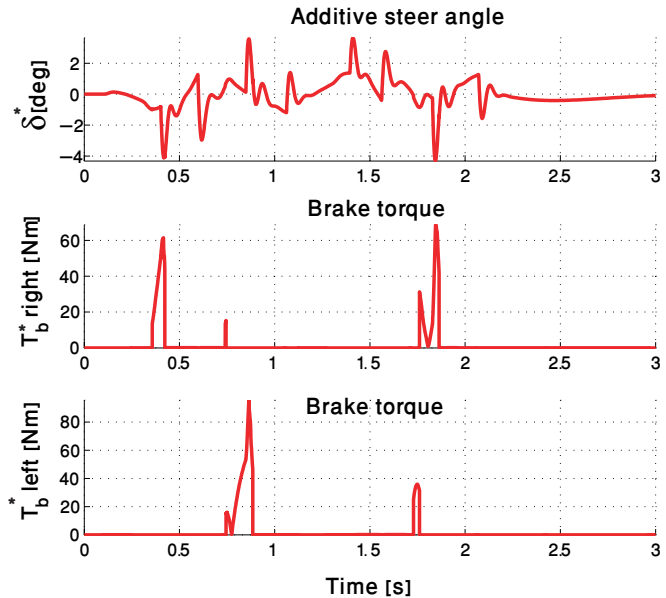

Fig. 9. Control signals generated by the controller for the double lane-change maneuver.

S. Anwar. Yaw stability control of an automotive vehicle via generalized predictive algorithm. Proceedings of the American Control Conference, Portland, USA, 2005.

B. L. Boada, M. J. L. Boada, and V. Diaz. Fuzzy-logic applied to yaw moment control for vehicle stability. Vehicle System Dynamics, volume 43, number 10, pages 753-770, 2005.

S. You, J. Hahn, and H. Lee. New adaptive approaches to real-time estimation of vehicle sideslip angle. Control Enginering Practice, volume 17, 1367-1379, 2009.

J. He, D. A. Crolla, M. C. Levesley, and W.J. Manning. Coordination of active steering, driveline, and braking for integrated vehicle dynamics control. Proc. IMechE, volume 220, PartD: Automobile Engineering, 2006.

X. Yang, Z. Wang, and W. Peng. Coordinated control of AFS and DYC for vehicle handling and stability based on optimal guaranteed cost theory. Vehicle System Dynamics, volume 47, number 1, pages 57-79, 2009.

C. Poussot-Vassal, O. Sename, L. Dugard, P. gaspar, Z. Szabo, J. Bokor. Attitude and handling improvements through gain-scheduled suspensions and brakes control. Control Engineering Practice (to appear).

H. Dugoff, P.S. francher, and L. Segel. An analysis of tire traction properties and their influence on vehicle dynamic performance. SAE transactions, volume 79, pages 341-366, 1970.

S. Skogestad and I. Postlethwaite. Multivariable feedback control, analysis and design, Wiley, 2007.

C. Scherer, P. Gahinet, and M. Chilali. Multiobjective output-feedback control via LMI optimization. IEEE Transaction on Automatic Control, volume 40, pages 896-911, 1997.

J. Lofberg. YALMIP: a toolbox for modeling and optimization in MATLAB. Proceedings of the CACSD Conference, Taipei, Taiwan, 2004.

J. F. Sturm. Using seDuMi 1.02, a Matlab toolbox for optimization over symmetric cones. Optimization Methods and Software 11-12, pages 625-653, Special issue on Interior Point Methods, 1999.

P. Apkarian and P. Gahinet. A convex characterization of gain scheduled $H_{\infty}$ controllers. IEEE Transaction on Automatic Control, volume 40, pages 853-864, 1995.

M. Doumiati, A. Victorino, D. Lechner, G. Baffet, and A. Charara. Observers for vehicle tyre/road forces estimations: experimental validation. Vehicle System Dynamics, volume 48(11), pages 1345-1378, 2010. 